\title{
Which patients and orthopedic material do get infected with Gram-negative non-fermenting rods?
}

\author{
I Uckay ${ }^{1 *}$, C Landelle², A Agostinho ${ }^{3}$, M Al-Mayahi $^{1}$, D Pittet $^{2}$ \\ From 3rd International Conference on Prevention and Infection Control (ICPIC 2015) \\ Geneva, Switzerland. 16-19 June 2015
}

\section{Introduction}

The $1^{\text {st }}$ and $2^{\text {nd }}$ generation cephalosporins used for perioperative prophylaxis do not cover non-fermenting Gram-negative rods (NFR).

\section{Objectives}

We investigated patient populations and types of surgery at risk for Gram-negative infections overall, and NFR in particular.

\section{Methods}

Retrospective cohort study of adult patients operated for orthopedic infections between 2004 and 2014. Only the first episode of infection was considered for analysis and infection diagnosis was based on intraoperative samples.

\section{Results}

The median age of all patients was 57 years; 871 were females, and 1021 were immune-suppressed. Overall, 665 episodes (24\%) involved osteosynthesis material (321 arthroplasties, 150 plates, 54 nails, and other materials). The median duration of antibiotic prescription prior to intraoperative sampling was 4 days; it occurred in $42 \%$ of all cases. Of the total 2740 surgical procedures, 568 grew Gram-negative pathogens (21\%) of which 258 (9\%) were NFR (120 episodes as co-infection) and 178 (7\%) Pseudomonas aeruginosa. Prior antibiotic use was significantly associated with NFR infections (159/947 vs. 99/1478; $p<0.01)$. On the median, NFR patients yielded 7 days of prior antibiotic use compared to 3 days of patients with non-NFR infections $(p<0.0001)$. Additional conditions associated with NFR infections were the presence of plates (25/144 vs. $233 / 2281 ; p<0.01)$ the presence of diabetic foot (57/385 vs. $201 / 2040 ; p<0.01$ ). Besides plate infection, no other hardware or prosthesis was particularly involved in
NFR infections. Risk was associated with an age older than 80 years. In this age category, NFR were responsible for $11 \%$ of all pathogens among orthopedic infections. In contrast, NFR were almost never documented in septic bursitis and less frequently associated with abscess formation in native bone or prosthetic joint infections. NFR infection was also less frequently identified in shoulder infections ( $3 / 80$ vs. $255 / 2345 ; p=0.04$ ).

\section{Conclusion}

The most important finding associated with orthopedic infections due to NFR is prior antibiotic use in elderly patients with diabetic foot infections/problems.

\section{Disclosure of interest}

None declared.

\section{Authors' details}

${ }^{1}$ Geneva University Hospitals, Geneva, Switzerland. ${ }^{2}$ Infection Control Program, Geneva University Hospitals, Geneva, Switzerland. ${ }^{3}$ Infection Control Program, Geneva University Hospitals, Geneva, Switzerland.

Published: 16 June 2015

\section{doi:10.1186/2047-2994-4-S1-P72}

Cite this article as: Uckay et al:: Which patients and orthopedic material do get infected with Gram-negative non-fermenting rods? Antimicrobial Resistance and Infection Control 2015 4(Suppl 1):P72.

${ }^{1}$ Geneva University Hospitals, Geneva, Switzerland

Full list of author information is available at the end of the article 